\title{
The relationship between work-life balance and quality of life among hospital employees
}

\author{
Roohollah Askari ${ }^{a}$, Sima Rafiei ${ }^{b}$, Rahele Akbari ${ }^{c}$, Elaheh Haghir Ebrahimi ${ }^{d}$, Arefeh Dehghani ${ }^{\mathrm{e}}$ and Milad Shafii ${ }^{\mathrm{a}}$ \\ ${ }^{a}$ Health Policy and Management Research Center, School of Public Health, Shahid Sadoughi University of Medical Sciences, Yazd, Iran; \\ ${ }^{b}$ Social Determinants of Health Research Center, Qazvin University of Medical Sciences, Qazvin, Iran; 'Department of Health Technology \\ Assessment, School of Public Health, Shahid Sadoughi University of Medical Sciences, Yazd, Iran; ${ }^{d}$ Health Care Management, School of \\ Public Health, Shahid Sadoughi University of Medical Sciences, Yazd, Iran; ${ }^{e}$ Biostatics and Epidemiology Department, School of Public \\ Health, Shahid Sadoughi University of Medical Sciences, Yazd, Iran
}

\section{ABSTRACT}

Introduction: Work-Life Balance (WLB) has got a significant attention among issues in a working environment. This study was conducted to investigate the current WLB status, and its' association with quality of life (QoL) among hospital employees.

Method: A cross-sectional survey of 210 hospital employees was conducted in a period of 3 months (April to June 2016) in three training hospitals in Yazd, Iran. Two standard questionnaires including WLB and WHO Quality of Life were used to gather data. The differences in the WLB by demographic variables were examined using Independent t-test and one-way ANOVA statistics. Pearson correlation coefficient was also used to examine the association between WLB and QoL among hospital employees.

Results: Most of the study participants devoted more time on work other than personal life. Employees' perception toward WLB was proved to be associated with QoL in all of the domains. However, there was no significant difference in terms of age, working shift, and work experience.

Conclusion: By increasing the WLB, QoL increases. Also, clinical staff and employed women were more likely to be the subject of poorer work-life balance which requires appropriate policy making in workplaces to effectively deal with the issue.

\section{ARTICLE HISTORY}

Received 20 May 2018

Accepted 2 August 2019

\section{KEYWORDS}

Work-life balance; quality of life; hospital; employee; relationship

\section{Introduction}

Recently, work-life balance (WLB) has got a significant importance among other issues in a working environment. The changing trend in working relationships, and recruitment rules over the past decades besides competitive pressures in modern organizations and growing business expectations have brought about a considerable increase in the amount of time which employees should spend in their workplace [1]. These changes have put the WLB in danger and emphasized on the necessity for well-designed policies to bring back this balance to work environments $[2,3]$.

WLB is referred to the existence of balance in the personal, family, and work life of individuals. It also explains the balance that an employee needs between time devoted to work and carry out other life programs. Overtime work duties and inflexible work shifts might cause a disparity between work and life duties. Failure to fulfill their family duties due to long working hours reduces family satisfaction, leads to stress, and eventually reduces the quality of life (QoL) among working individuals. As cited by Kofodimos (1993), imbalance is recognized as 'a problem that mainly harms individual's life quality and well-being' [4]. Generally QoL can be improved by making balance between work and private life. QoL mirrors a person's expectations for a desirable life. These expectations come from the socio-cultural context and value system of the society in which people live in [5]. This multidimensional concept identifies a standard level for physical, mental, social and environmental well-being. In fact, establishing WLB can lead to better performance, greater motivation, lower absenteeism, and reduced psychological disorders among employees [3].

Recent research has shown that $70 \%$ of employees were dissatisfied with their WLB, and half of them were experiencing high levels of stress and discomfort. These negative effects not only imposed additional costs to organizations, but also decreased the effectiveness in achieving organizational objectives [6]. In a study conducted by Gallup in 2001, findings revealed that $5 \%$ of employees were under stress in their workplace due to experiencing some levels of work-life imbalances. The study results also mentioned an increase in absenteeism, low productivity, high rates of physical and mental illnesses among employees as some of the main undesirable consequences of this

CONTACT Milad Shafii $\otimes$ milad.shafii@gmail.com $=$ Department of Health Services Management, School of Public Health, Shahid Sadoughi University of Medical Sciences, Yazd 891517316, Iran

(c) 2019 Informa UK Limited, trading as Taylor \& Francis Group 